\title{
Amblyomma aureolatum (PALLAS, 1772) PARASITANDO CAPIVARA (Hidrochaeris hidrochaeris) NA REGIÃO DO PLANALTO CATARINENSE-RELATO DE CASO
}

Recebido: 30/11/2014

QUADROS, Rosiléia Marinho de ${ }^{1}$; MARQUES, Sandra Márcia Tietz ${ }^{2}$; VERONEZI, Wilian Rafael ${ }^{3}$; CARNEIRO JÚNIOR, Jary André ${ }^{4}$.

Aceito: 10/06/2015

${ }^{1}$ Médica Veterinária, Bióloga, Professora, Doutora, Centro de Ciências Agroveterinárias, UDESC e UNIPLAC;

${ }^{2}$ Médica Veterinária, Doutora, Faculdade de Veterinária, UFRGS; ${ }^{3}$ Biólogo, IBAMA/Lages/SC; ${ }^{4}$ Biólogo, Laboratório de Parasitologia, UNIPLAC.

\section{RESUMO}

\begin{abstract}
A mblyomma aureolatum apresenta como hospedeiros primários os carnívoros, enquanto as larvas e ninfas parasitam passeriformes e roedores. Este é o primeiro relato que descreve Hidrochaeris hidrochaeris como hospedeiro primário de $A$. aureolatum.
\end{abstract}

Palavras-chave: Ectoparasitos. Ixodidade. Hospedeiros.

\section{INTRODUÇÃO}

A capivara (Hidrochaeris hidrochaeris Linnaeus, 1766) é o maior roedor do mundo. Seu habitat natural envolve locais de pastagens, matas ciliares, manguezais, banhados e locais com presença de corpos d’água (MOREIRA; MACDONALD, 1997). É um herbívoro semiaquático com distribuição na América Central e do Sul (exceto Chile), do Panamá ao nordeste da Argentina (EISENBERG; REDFORD, 1999).

Existem cerca de 870 espécies de carrapatos descritas no mundo e aproximadamente 60 relatadas no Brasil (BARROS-BATTESTI et al., 2006). A superfamília Ixodoidea compreende três famílias: Argasidae e Ixodidae com ampla distribuição geográfica e a família Nuttalliellidae que ocorre mais confinada a um número reduzido de continentes (KEIRANS et 
al., 1976). O gênero Amblyomma apresenta aproximadamente 130 espécies, 57 descritas na região neotropical e 33 no Brasil (GUIMARÃES et al., 2001; LABRUNA, 2004).

Amblyomma aureolatum apresenta como hospedeiros primários os carnívoros, enquanto as larvas e ninfas parasitam passeriformes e roedores (Caviomorfos) (LABRUNA et al., 2001). O

ciclo biológico das espécies $A$. cajennense, $A$. aureolatum e A. dubitatum exige três hospedeiros para completá-lo. Em animais silvestres no sul do Brasil, como hospedeiros primários, foram registrados em Didelphis albiventris (gambá) em Pelotas (RS) (MULLER et al., 2005); Alouatta guariba (bugio) em Cachoeira do Sul (RS) (MARTINS et al., 2006); Leopardus wiedii (gato-maracajá) em Gravataí (RS) (MARTINS et al., 2010); Alouatta clamitans na região norte do estado de Santa Catarina (LAVINA et al., 2011) e carnívoros silvestres como Puma concolor (puma), Puma yagouroundi (gato mourisco), Cerdocyon thous (cachorro do mato) e Procyon cancrivorus (mão-pelada) no Planalto Catarinense (QUADROS et al., 2013). O objetivo deste relato é registrar a presença de $A$. aureolatum em um roedor como hospedeiro primário.

\section{RELATO DE CASO}

Capivara macho jovem da espécie $H$. hidrochaeris proveniente da localidade rural de Coxilha Rica no município de Lages, planalto serrano do estado de Santa Catarina foi resgatado no ambiente rural e introduzido em outubro de 2012 na Base Avançada (BAP) do Instituto Brasileiro de Meio Ambiente (IBAMA) no município de Painel (SC). A BAP está localizada junto à rodovia SC $114 \mathrm{~km} 242\left(27^{0} 52^{\prime} 12^{\prime \prime} \mathrm{S}\right.$ e 62 $\left.11^{\prime} 47^{\prime \prime} \mathrm{W}\right)$. O animal aparentemente era jovem e apresentava boa condição corporal, com peso aproximado de $30 \mathrm{~kg}$. Antes da soltura, o animal foi examinado, observando-se a presença de um carrapato da família Ixodidae na região ventral. O ácaro foi coletado com auxílio de uma pinça e preservado em álcool 70\%. A identificação foi realizada com uso da chave segundo Barros-Battesti et al. (2006), com auxílio de estereoscópio no Laboratório de Parasitologia do Centro de Ciências Agroveterinárias da Universidade do Estado de Santa Catarina (CAV-UDESC). O ixodídeo foi depositado na coleção zoológica do Laboratório de Parasitologia da Universidade do Planalto Catarinense (UNIPLAC). 


\section{RESULTADOS E DISCUSSÃO}

$\mathrm{Na}$ comparação do exemplar com as informações contidas na chave (BARROS-BATTESTI et al., 2006), a espécie identificada foi $A$. aureolatum (macho). O espécime que tem como características essenciais para identificação a presença, na coxa 1, de espinhos longos, subiguais, contíguos e muito longos, o externo terminando em ponta reta; o escudo é castanho-claro com predomínio do amarelo-dourado; o sulco marginal incompleto, alcançando até o segundo festão (Figura1) e hipostômio com dentição 3/3
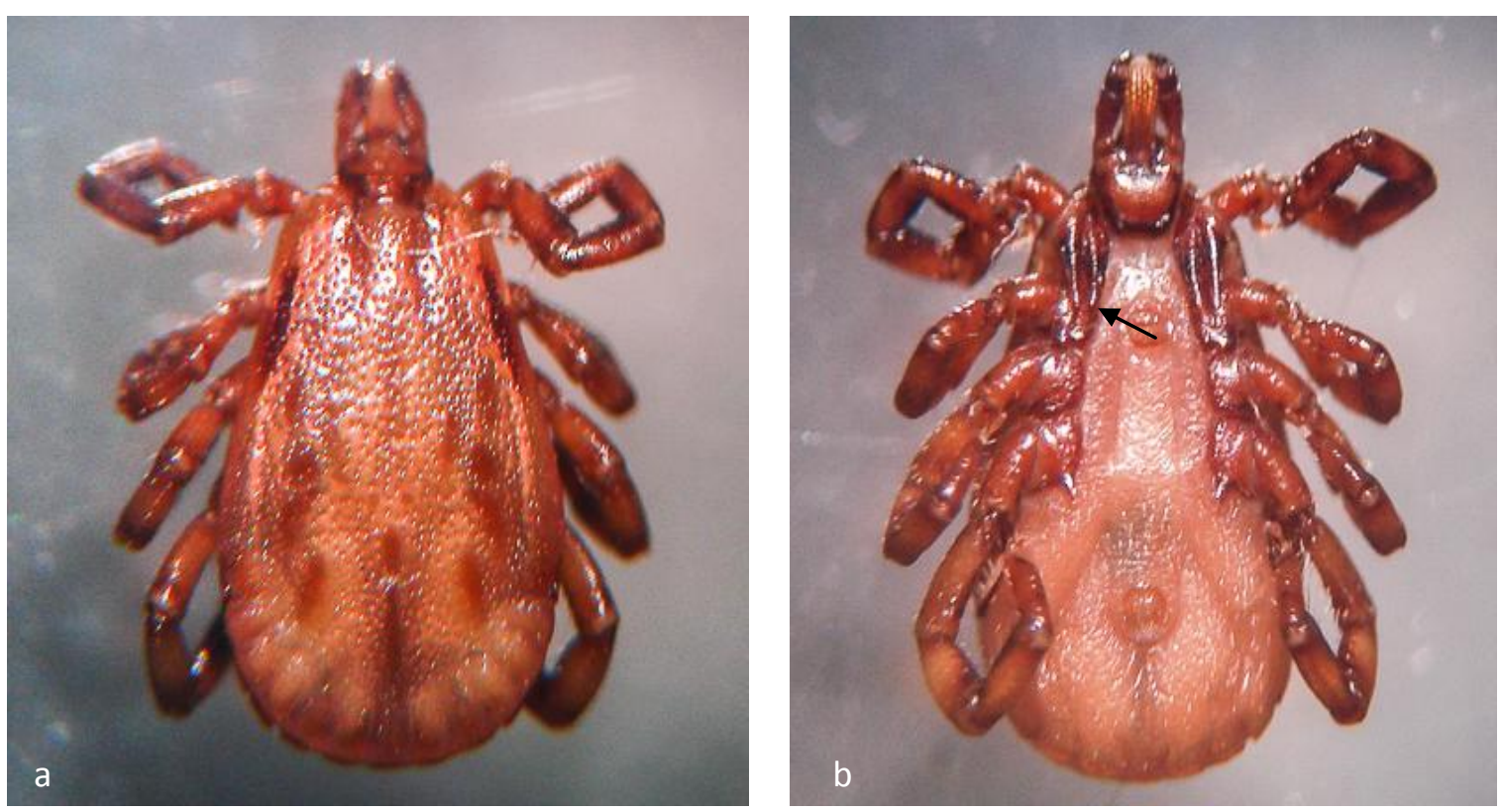

Figura 1 - Características morfológicas do macho: vista dorsal (a) e ventral (b). Seta: coxa 1 com espinho longo e desigual.

Amblyomma aureolatum parasita carnívoros na fase adulta e aves e roedores na fase imatura (BARROS-BATTESTI et al., 2006). O achado do espécime adulto em capivara registra que é possível que $A$. aureolatum tenha outros hospedeiros primários além de carnívoros. Segundo Ribeiro (1970) é a espécie mais frequente em cães no estado do Paraná; e no Brasil tem registros nos estados do Rio Grande do Sul, Santa Catarina, Paraná, São Paulo, Rio de Janeiro, Minas Gerais, Pernambuco, Bahia, Piauí e Sergipe (ARZUA et al., 2003). Ainda que tenha sido registrado em áreas quentes do Brasil, este carrapato ocorre principalmente nos 
cães que frequentam áreas rurais das regiões Sul e Sudeste, juntamente com $R$. sanguineus e outras espécies de Amblyomma (LABRUNA et al., 2001). Em Santa Catarina, foi registrado pela primeira vez parasitando Puma concolor com espécimes ninfas e adultos (MARQUES et al., 2010) e nas regiões sul e grande Florianópolis foram coletados em cães (LAVINA et al., 2014). No estado do Rio Grande do Sul foi registrado parasitando cães, gatos, graxaim e mão-pelada (EVANS et al., 2000).

O espécime é vetor da febre maculosa brasileira (FMB), uma doença que acomete humanos, infecciosa, febril, aguda, de gravidade variável, cuja apresentação clínica pode variar desde formas leves e atípicas até formas graves, com elevada taxa de letalidade. É causada por uma bactéria do gênero Rickettsia (Rickettsia rickettsii) transmitida por carrapatos e tem sido registrada em áreas rurais e urbanas do Brasil. A maior concentração de casos é verificada nas regiões Sudeste e Sul, onde de maneira geral ocorre de forma esporádica. Quanto à sazonalidade, verifica-se que o período de maior incidência é em outubro, período no qual se observa maior densidade de ninfas de carrapatos, podendo variar de região para região. A FMB foi incluída na Lista Nacional de Doenças de Notificação Compulsória do Ministério da Saúde, pela Portaria GM/MS no 1.943, de 18 de outubro de 2001 (SVS, 2009).

Os relatos da transmissão da febre maculosa no Brasil apontam os carrapatos do gênero Amblyomma como sendo o principal vetor. Os carrapatos permanecem infectados durante toda a vida, em geral de 18 a 36 meses. Essa infecção pode ser propagada para outros carrapatos por meio da transmissão vertical (transovariana), da transmissão estádio-estádio (transestadial) ou da transmissão através da cópula, além da possibilidade de alimentação simultânea de carrapatos infectados com não infectados, em animais com suficiente riquetsemia. Entretanto, potencialmente, qualquer espécie de carrapato pode ser reservatório, como ocorre com o Haemaphysalis leporispalustris (carrapato do coelho). Os equídeos e roedores, como a capivara (Hydrochaeris hydrochaeris) e marsupiais, como o gambá (Didelphis sp.), têm importante participação no ciclo de transmissão da febre maculosa e há estudos recentes sobre o envolvimento desses animais como reservatórios ou amplificadores da Rickettsia, assim como transportadores de carrapatos potencialmente 
infectados. Conhecer a biologia do carrapato é importante para a implementação de medidas de controle eficazes e oportunas (SVS, 2009).

O gênero Amblyomma tem características próprias, como a sua grande resistência no meio ambiente, não requer especificidade de hospedeiro, portanto pode alimentar-se em equídeos, bovinos, caprinos, homens, aves silvestres, cães, gatos, entre outros. A partir da identificação das áreas de risco e ocorrência comprovada de casos da febre maculosa, a vigilância epidemiológica deve atuar ativamente, para evitar novas ocorrências. As áreas de risco relacionam-se, principalmente, com a presença e disponibilidade dos vetores e dos reservatórios (SVS, 2009).

No Sul do Brasil, A. aureolatum é uma das espécies que melhor se adapta ao cão, no qual se apresenta com grande frequência nas regiões habitadas pelo homem. Também é encontrado com regularidade em animais silvestres de áreas frequentadas por cães.

\section{CONCLUSÃO}

A identificação de $A$. aureolatum parasitando capivaras (H. hidrochaeris) justifica o presente relato, uma vez que o roedor ocorre cada vez mais na região do Planalto Catarinense e compartilha ambientes frequentados por diversas espécies de animais silvestres.

\section{NATURAL INFESTATION OF CAPYBARA (Hidrochaeris hidrochaeris) WITH Amblyomma aureolatum (PALLAS, 1772) IN THE PLATEAU REGION OF SANTA CATARINA, BRAZIL-CASE REPORT}

\section{ABSTRACT}

$\mathrm{C}$ arnivorous are the definitive hosts of Amblyomma aureolatum; however, its larvae and nymphs infest birds and rodents. This report describes the first case of Hidrochaeris hidrochaeris as the primary host of A. aureolatum.

Keywords: Ectoparasites. Ixodidae. Hosts. 


\section{Amblyomma aureolatum (PALLAS, 1772) PARASITANDO CAPIBARA (Hidrochaeris hidrochaeris) EN LA REGIÓN DEL PLANALTO CATARINENSE- RELATO DE UN CASO}

\section{RESUMEN}

A mblyomma aureolatum presenta como hospedadores primarios los carnívoros, mientras larvas y ninfas parasitan paseriformes y roedores. Este es el primer relato que describe por primera vez Hidrochaeris hidrochaeris como hospedador primario de A. aureolatum.

Palabras clave: Ectoparasitos. Ixodidade. Hospedadores.

\section{REFERÊNCIAS}

ARZUA, M.; NAVARRO DA SILVA, M. A.; FAMADAS, K. M.; et al. Amblyomma aureolatum and Ixodes auritulus (Acari: Ixodidae) on birds in Southern Brazil, with notes on their ecology. Experimental and Applied Acarology, v. 31, n. 3-4, p. 283-296, 2003.

BARROS-BATTESTI, D. M.; ARZUA, M.; BECHARA, G. H. Carrapatos de importância médicoveterinária da Região Neotropical: um guia ilustrado para identificação de espécies. São Paulo: Vox-ICTTD-3-Butantan, 2006. 223p.

EISENBERG, J. F.; REDFORD, K. H. Mammals of the Neotropics: the central neotropics. Chicago: University of Chicago, 1999. 609p.

EVANS, D. E.; MARTINS, J. R.; GUGLIELMONE, A. A. A review of the ticks (Acari, Ixodida) of Brazil, their hosts and geographic distribution - 1. The State of Rio Grande do Sul, Southern Brazil. Memórias do Instituto Oswaldo Cruz, v. 95, n. 4, p. 453-470, 2000

GUIMARÃES, J. H.; TUCCI, H. E. C.; BARROS-BATTESTI, D. M. Ectoparasitos de importância veterinária. São Paulo: Plêiade, 2001. 213p.

KEIRANS, J. E.; CLIFFORD, C. M.; HOOGSTRAAL, H.; et al. Discovery of Nuttallie Illanamaqua Bedford (Acarina: Ixodoidea: Nuttalliellidae) in Tanzania and redescription of the female based on scanning electronmicroscopy. Annals of the Entomological Society of America, v. 69, p. 926-932, 1976.

LABRUNA, M. B.; SOUZA, S. L. P.; GUIMARÃES, J. S.; PACHECO, R. C.; PINTER, A.; GENNARI, S. $M$. Prevalência de carrapatos em cães de áreas rurais da região norte do Estado do Paraná. Arquivo Brasileiro de Medicina Veterinária e Zootecnia, v. 53, n. 5, 2001. 
LABRUNA, M. B. Carta acarológica. In: CONGRESSO BRASILEIRO DE PARASITOLOGIA VETERINÁRIA E SIMPÓSIO LATINO-AMERICANO DE RICKETTSIOSES, 13, 2004, Ouro Preto. RESUMOS. Belo Horizonte: CBPV, 2004, p. 199-202.

LAVINA, M. S.; SOUZA, A. P.; BELLATO, V.; SARTOR, A. A.; MOURA, A. B. Ocorrência de Amblyomma aureolatum (Pallas, 1772) e A. ovale (Kock, 1844) (Acari: Ixodidae) parasitando Alouatta clamitans (Cabrera, 1940) (Primates: Atelidae) na região norte do estado de Santa Catarina. Arquivo Brasileiro de Medicina Veterinária e Zootecnia, v. 63, n. 1, p. 266-269, 2011.

LAVINA, M. S.; SOUZA, A. P.; BELLATO, V.; et al. Ixodídeos coletados em equinos e caninos no Estado de Santa Catarina. Revista Brasileira de Medicina Veterinária, v. 36, n. 1, p. 1-6, 2014.

MARQUES, S. M. T.; QUADROS, R. M.; MAZZOLLI, M.; et al. Amblyomma aureolatum (Pallas, 1772) (Acari: Ixodidae) em Puma concolor: primeiro registro em Santa Catarina, Brasil. Veterinária em Foco, v. 7, n. 2, p. 148-152, 2010.

MARTINS, J. R.; RECK JÚNIOR, J.; DOYLE, R. L.; CRUZ, N. L. N.; VIEIRA, A. W. M.; SOUZA, U. A. Amblyomma aureolatum (Acari: Ixodidae) parasitizing margay (Leopardus wiedii) in Rio Grande do Sul / Amblyomma aureolatum (Acari: Ixodidae) parasitando Gato-Maracajá (Leopardus wiedii) no Rio Grande do Sul. Revista Brasileira de Parasitologia Veterinária, v. 19, n. 3, p. 189-191, 2010.

MARTINS, J. R.; SALOMÃO, E. L.; DOYLE, R. L.; TEIXEIRA, M. C.; ONOFRIO, V. C.; BARROSBATTESTI, D. First Record of Amblyomma aureolatum (Pallas, 1772) (Acari: Ixodidae) parasitizing Alouatta guariba (Humboldt, 1812) (Primata: Atelidae) in southern Brazil. Revista Brasileira de Parasitologia, v. 15, n. 4, p. 203-205, 2006.

MOREIRA, J. R.; MACDONALD, D. W. Técnicas de manejo de capivaras e outros grandes roedores na Amazônia. In: VALLADARES-PADUA, C.; et al. (Eds.). Manejo e conservação de vida silvestre no Brasil. Belém: Sociedade Civil Mamiraúa, 1997, p. 186-213.

MULLER, G.; BRUM, J. G. W.; LANGONE, P. Q.; MICHELS, G. H.; SINKOC, A. L.; RUAS, J. L.; BERNE, M. E. A. Didelphis albiventris (Lund, 1841) parasitado por Ixodes loricatus (Neumann, 1899) e Amblyomma aureolatum (Pallas, 1772) (Acari: Ixodidae) no Rio Grande do Sul. Arquivos do Instituto Biológico, v. 72, n. 3, p. 319-324, 2005.

QUADROS, R. M.; BOAVENTURA, B. L.; VERONEZI, W.; MARQUES, S. M. T. Fauna de ixodídeos em carnívoros silvestres atropelados em rodovias de Santa Catarina: Relato de caso.

Veterinária em Foco, v. 10, n. 2., p. 222-228, 2013.

RIBEIRO, S. S. Ixodídeos encontrados no cão doméstico no estado do Paraná. Anais da Faculdade de Medicina, Universidade Federal do Paraná, v. 13-14, n. 1-2, p. 61-67, 1970. 
SVS-SECRETARIA DE VIGILÂNCIA EM SAÚDE /MS. Febre Maculosa Brasileira. Guia de Vigilância Epidemiológica. 7. Ed. Brasília. DF. MS. 2009. 816p. Disponível em:

http://bvsms.saude.gov.br/bvs/publicacoes/guia_vigilancia_epidemiologica_7ed.pdf. Acesso em: 20 out. 2014.

Autor para correspondência: Rosiléia Marinho de Quadros. Centro de Ciências Agroveterinárias da Universidade do Estado de Santa Catarina (CAV/UDESC), Avenida Luiz de Camões, 2090, Lages (SC), CEP 88520-000. rosileia18@hotmail.com 